\title{
EL HUAYNO EN LA CIUDAD: \\ LA MÚSICA ANDINA EN EL SEXTO
}

\author{
POR \\ JoRge CORONADO \\ Northwestern University
}

¿Qué se aprende desde la música en los textos de José María Arguedas? Es decir, ¿en qué forma y hasta qué punto se puede entender la música como una propuesta para un modelo interpretativo más allá de su capacidad representacional o simbólica? Por ello no es mi intención minimizar la importancia de su uso como símbolo en la obra arguediana: de hecho, la música y sus avatares acaso sean las figuras predilectas para la expresión y difusión de los conceptos que subyacen de manera fundamental en su narrativa: la indigeneidad y el mestizaje. La música andina se encarga de esa labor representacional sustancial en Arguedas, aunque lo comparta con los aparatos significadores que son la naturaleza, el idioma quechua, y la niñez, entre otros. Sin embargo, con la música Arguedas excede la mera representación e imagina una máquina de interpretación. Esta máquina aparece como respuesta a los desafíos de la historia y sociedad coloniales en los Andes, pero también, y acaso con más fuerza, para entender el lugar y la función de la cultura andina en la modernidad. En un artículo reciente, el crítico Jon-Beasley Murray propone que siempre hay una máquina en las novelas de Arguedas, y que ésta funciona para efectuar "the conversion of one form of energy into another." Habla explícitamente de la guitarra en El Sexto como un ejemplo (123). Aunque no seguiré su línea de argumentación respecto al "affect" en la obra arguediana, me parece útil la idea que estas máquinas en Arguedas sirven para fabricar una modernidad andina.

La pregunta surge en un contexto particular, que será el enfoque de este artículo. La novela El Sexto se asemeja a y se distingue de las otras novelas de Arguedas, y acaso se parezca más a El zorro de arriba y el zorro de abajo que a cualquier otra. Como anuncia la portada del libro, Arguedas comenzó a escribirla en 1957 luego de idearla en 1939. La publica en 1961, entre la aparición de Los ríos profundos en 1958 y Todas las sangres en 1964. El Sexto pues forma parte de un momento fecundo en la producción arguediana y reitera la lucha del hombre y la cultura andina por hacerse lugar en y adueñarse de la modernidad. Sin embargo, en El Sexto esa lucha se da dentro de la encarnación del aparato ideológico opresivo, se denomine hispanismo, 
criollismo, capitalismo, o con otro rótulo. Me referiré a ese conjunto con los términos que intercambiablemente utiliza en esta novela el mismo Arguedas para los poderes opresores: Lima y el Sexto.

La música ha sido un enfoque central o pasadero de un sinnúmero de estudiosos de la obra de Arguedas. Entre las intervenciones críticas destacadas sobre el tema se incluyen las de Antonio Cornejo Polar, Ángel Rama, Martín Lienhard, William Rowe, y otros. En verdad, hay pocos críticos que no hayan tomado en cuenta el significado y la potencia de la música andina en esta obra. Son contados, sin embargo, los que han trabajado la música en su conexión a la modernidad urbana y específicamente al moldeamiento de la música andina dentro de las especificidades del momento histórico en que Arguedas escribe. Rowe, en sus Ensayos arguedianos, sugiere convincentemente que la reflexión sobre la música en Arguedas no se puede limitar a sus textos narrativos y debe incluir sus trabajos de vertiente antropológica y etnográfica, algunos de los cuales se encuentran en la obra periodística (36). Precisamente, ésta representa un punto clave, puesto que nos permite entender una obra letrada cuyas partes diversas se complementan e iluminan. Para el presente trabajo, la conjunción de la obra periodística y la novelesca será particularmente reveladora.

Rowe sugiere que la música se debe interpretar como un "ordenamiento de las percepciones y contribución a la producción de un lugar de enunciación" (36). En las décadas de los cincuenta y los sesenta, Arguedas se vio como actor principal en la aparición de la música andina tradicional en la ciudad. Esta parte de su obra nos aportará pistas para entender cómo funciona la música en $E l$ Sexto, que se escribe coetáneamente. Aunque a esta novela no le toca demostrar la construcción de un lugar de enunciación que permitiría articular lo andino con la modernidad, creo que la forma definitiva, futura, de ese lugar, o por lo menos el mecanismo que llevaría a él, queda claramente señalada. Se trata pues de un paso hacia la fabricación de un lugar enunciativo.

Quisiera ampliar el análisis de Rowe respecto a la música en sus Ensayos arguedianos, puesto que la preponderancia del mercado de consumo en El Sexto y Los zorros, bien entendido en formas distintas, requiere pensar en la concretización local del fenómeno del capitalismo global. Rowe sugiere que la elaboración de una representación musical en Arguedas se debería considerar como un espacio sonoro dentro del cual "todo puede ser oído" (45). Rowe entiende una evolución progresiva en Arguedas hacia esta posibilidad, que se realiza en Los zorros. Se trata de un enfoque que identifica lo comunicativo con lo sonoro en Arguedas, pues Rowe propone que la música representa esa posibilidad y que se elabora con esa meta. El Sexto se debe entender como parte de esta estrategia, sin duda. Pero argüiría que en ese texto, el espacio para el entendimiento de la música es mucho más hostil que en las novelas de ambientación serrana, como Yawar fiesta o Todas las sangres. De alguna forma, en El Sexto ese espacio resulta controvertido y conflictivo, pues así también es la ciudad

Revista Iberoamericana, Vol. LXXXI, Núm. 253, Octubre-Diciembre 2015, 1051-1064 
costeña que ya está llena de música de otras estirpes. Esas músicas suelen ubicarse en el mercado real y simbólico. El mercado determina lo que la música puede ser en la prisión así como también lo hace en el espacio urbano más allá de ella.

Por último, habría que corregir una impresión a mi ver equívoca que tiene la crítica en general: que El Sexto es una novela rara, inconexa, o menor en la obra de Arguedas. $\mathrm{Al}$ contrario, y visto desde la óptica de la narrativa y la ensayística arguedianas como una totalidad, El Sexto tiene un papel fundamental precisamente porque constituye el primer tratamiento no de la modernidad, pues eso está en juego desde los primeros textos, sino más bien de la complejidad del ambiente urbano y lo que éste implica en los procesos culturales andinos.

La novela sigue la reclusión del personaje central, Gabriel, en la cárcel citadina. Gabriel es estudiante de origen serrano, y al parecer por la reacción de los presos que lo reciben, ya se lo reconoce como intelectual y probablemente escritor. Pero El Sexto no se preocupa prioritariamente por el desarrollo o evolución de ese personaje, sino más bien por retratar la sociedad que contiene la cárcel. En ella, se encuentran por lo menos tres grupos distintos de presos que se organizan espacialmente según los tres pisos del Sexto. En el primero habitan los criminales comunes avezados, entre los cuales se encuentran asesinos, ladrones, etc., pero también los llamados vagos, que pululan en masa y parecen haber perdido el uso de la razón por los abusos que han sufrido; en el segundo piso habita un surtido de encarcelados no avezados, harto variados entre ellos; en el tercero, se encuentran los prisioneros políticos, que pertenecen a dos partidos, el APRA y el comunista. Entre éstos existe conflicto sobre todo ideológico, pero se mantiene una especie de tregua entre ellos. Gabriel, que no asume una identidad política de partido ni se pronuncia como adherente, comparte celda en este piso. Al ser categorizado como "estudiante" por los grupos políticos, se entiende que Gabriel ocupa un espacio que podríamos llamar pre-ideológico, en el contexto de la política nacional. Efectivamente, Gabriel mantiene cierta distancia de la ideología política rígida de partido tal como la expresan los cabecillas.

Más allá de los elementos descriptivos con respecto a la vida carcelaria y los personajes que allí se encuentran, la trama de la novela consistiría entonces en la articulación de un lugar cultural e ideológico desde el cual se podría escapar de la fragmentación y deterioro sociales que caracterizan el Sexto. Es decir, y de acuerdo a la descripción que hemos hecho del espacio carcelario y la sociabilidad (o falta de ella) a la que lleva, en El Sexto se propone mostrar la división que la sociedad peruana dominante y su estado generan, apoyan y propagan, para luego proponer una solución. Como dice Antonio Cornejo Polar, la institución carcelaria aquí "revela [...] el macrocosmos nacional" (154). Para ello, resulta necesario que la novela se concentre en dos temas centrales a través de las dinámicas entre los presos: la inhumanidad del hombre para con sus prójimos y la imposibilidad de la comunicación a través del discurso racional.

Revista Iberoamericana, Vol. LXXXI, Núm. 253, Octubre-Diciembre 2015, 1051-1064 
El rechazo de esas dos sociabilidades malsanas permite postular una tercera opción, que emerge en varios aspectos de la vida carcelaria pero en ninguno tanto como en la que se cifra a través de la música, y la musicalidad, andinas. Vale la pena notar que esa tercera opción toma forma a través de las interacciones de un grupo de serranos que exceden los límites de cada piso, pues están distribuidos en todos. Estos tienen por lo menos un factor en común: son provincianos, es decir, no pertenecen a Lima ni a su cultura. Opera pues una fuerte oposición entre costa y sierra en la matriz de la novela. Esta oposición resulta uno de los lugares comunes de la producción letrada andina del siglo XX. En cierto grado, Arguedas la resalta para luego derribarla.

Según el crítico peruano Alberto Escobar, en la novela se encuentra un esbozo preparativo para la novela póstuma. En algo tiene razón. El parecido sin duda se debe al entorno urbano. Como Los zorros, se trata de una narrativa que se despliega enteramente en la costa; no como la otra novela, El Sexto se sitúa en el mismo centro, literal y figurativamente, de Lima, ubicación que le infunde el significado pleno de su historia y su cultura. Más que la capital nacional, representa la fuente y el centro radiantes del criollismo y del colonialismo, concretización en la urbanización de aquello que Sebastián Salazar Bondy designó el nacionalismo limeño. Es pues, como diría el mismo Arguedas en su poema "A nuestro padre creador Tupac Amaru", un texto que se enfrenta de forma abierta, directa, con "la cabeza corrompida de los falsos wiraqochas" (Katatay 17). Quizás esté demás decirlo, pero sería imposible situar Los zorros en la ciudad de los virreyes, precisamente porque el desarrollo explosivo de Chimbote se da como un fenómeno excéntrico al nacionalismo limeño y a su vez céntrico al capitalismo global que produce la sociedad y sociabilidad movedizas y los hervores identitarios que caracterizan esa novela. En Los zorros el problema consiste, visto en grueso, en entender las posibilidades de los Andes y sus culturas en los flujos socioeconómicos globales. En El Sexto, por el contrario, se trata de responder a través de la formulación de un modelo de sobrevivencia y acaso de superación, ante la estructura social vigente, decimonónica y persistentemente colonial. Si se me permite la simplificación, Los zorros ve la posibilidad futura de la Historia (con mayúscula); El Sexto brega con el peso lúgubre de la historia hasta el momento de su elaboración. En ambas, la ciudad asume un papel central, pero las ópticas que se elaboran desde ella dependen tanto de las reglas sociales que la rigen -endurecidas en Lima y flexibles en Chimbote- como de la potencia u opositora o absorbente de la cultura andina en esos espacios. No cabe duda de que la cultura andina cifrada como música serrana se transforma en opción en El Sexto porque resulta ser, como si por esencia, una fuerza opositora.

En la ciudad, y sobre todo en los mercados de consumo que alberga, se gesta una de las razones principales por la potencialidad de la música tal como se representa en El Sexto. Al fin y al cabo, la novela opera bajo la influencia decisiva de una crítica al consumo y la producción de bienes dentro del modo capitalista. Esta crítica se encuentra en las diatribas del indígena comunista Cámac, como por ejemplo cuando juzga:

Revista Iberoamericana, Vol. LXXXI, Núm. 253, Octubre-Diciembre 2015, 1051-1064 
De esos gringos que he visto en Morococha no lo creo, compañero. Uno que tiene a su padre y a su madre y a su patria y va a otra nación para hacer millones con la sangre y la tierra extranjera, acaso, si es hombre criado por padres y madres, ¿puede escupir al trabajador que le hace ganar millones? ¿Puede escupirlo? ¡Ahistá! Ese no tiene crianza. Por eso, como maldición, no hay para él otro apoyo que las balas. ¡Balas y billetes, es la patria del gringo! (El Sexto 24)

La música andina pues se encarga de responder a una economía extractiva tal como la describe Cámac. Nótese que el efecto práctico de este tipo de economía y los mercados que la generan es el desarraigo nacional, es decir, el sustituir una patria geopolítica con la ganancia y la violencia. Precisamente estos dos elementos definirán no sólo lo maligno del microcosmos carcelario, sino también del nacionalismo criollo.

Antes de destilar lo que debería ser una música transcendente y purificadora en oposición al ensuciamiento del Sexto, Arguedas elabora una perspectiva mucho más amplia respecto a los usos y tergiversaciones de la música en cuanto aparato cultural. Esa perspectiva abarca más que sus elaboraciones al respecto que se encuentran en sus otras novelas, aunque las incluye también. En El Sexto la música tiene una función fundamentalmente distinta, aunque asociada, a la que asume en obras como Los ríos profundos, por ejemplo. Esencia de la naturaleza para el niño Ernesto y lugar figurativo de encuentro entre tradiciones andinas divergentes, la expresión musical -y allí sí podemos hablar del huayno que domina- en ese texto se encuentra rodeada por las comunidades humanas que de alguna forma la originan y además está presente en todo aspecto de la sociedad circundante: tanto mistis como cholas y colonos, y, además, andinos de regiones variadas, la comparten aunque, claro está, con formas de recepción distintas. Baste una descripción de las costumbres en la ciudad serrana de Abancay que se encuentra en Los ríos profundos:

Las chicherías recibían gente desde el mediodía, pero sólo en la tarde y en la noche de los sábados y domingos iban los músicos. Cualquier parroquiano podía pedir que tocaran el huayno que prefería. Era difícil que el arpista no lo supiera. A las chicherías van más forasteros que a un tambo. Pero ocurría, a veces, que el parroquiano venía de tierras muy lejanas y distantas; de Huaraz, de Cajamarca, de Huancavelica o de las provincias del Collao, y pedía que tocaran un huayno completamente desconocido. Entonces los ojos del arpista brillaban de alegría; llamaba al forastero y le pedía que cantara en voz baja. Una sola vez era suficiente. El violinista lo aprendía y tocaba; el arpa acompañaba. Casi siempre el forastero rectificaba varias veces: “¡No; no es así! ¡No es así su genio!’ Y cantaba en voz alta, tratando de imponer la verdadera melodía. Era imposible. El tema era idéntico, pero los músicos convertían el canto en huayno apurimeño, de ritmo vivo y tierno. “Mánan!” gritaban los hombres que venían de las regiones frías; los del Collao se enfurecían, y si estaban borrachos, hacían callar a los músicos amenazándolos con los grandes vasos de chicha. "Igual es, señor," protestaba el arpista. “¡No, alk’o (perro)!” vociferaba el collavino. Ambos tenían razón. (209)

Revista Iberoamericana, Vol. LXXXI, Núm. 253, Octubre-Diciembre 2015, 1051-1064 
El desacuerdo que surge del encuentro entre diferentes versiones musicales, que aquí no se pueden separar de la naturaleza tal como se da en la región de origen de cada versión, no lleva a la disolución social como sí es el caso en El Sexto. Al contrario, aquí se transforma en la ocasión para una especie de hibridación, conflictiva sí, pero feliz y oportuna, pues el producto cultural mezclado permite una sociabilidad nueva y mayor. En Los ríos, en Yawar fiesta, en Todas las sangres, la música andina asume el papel de un patrimonio común que no logra crear una comunidad igualitaria y ética, pero que sin embargo sigue siendo en esas novelas el lugar desde el cual las conexiones profundas que subyacen a la diversidad de sujetos sociales se pueden constatar. Esta función se importa a El Sexto.

Volviendo a esa novela, la música se reproduce en manifestaciones múltiples que incluyen himno político, tango, muliza, vals criollo, y claro, huayno. Esta música andina se da en formas que compiten entre muchas otras o más bien, buscan distinguirse de los usos negativos que encuentran esos otros géneros musicales en la cárcel: la escisión política y la violencia sexual, ambas representadas como métodos que hacen imposible la humanidad del hombre, vale decir también, la peruanidad del hombre andino. Se señala la solución a menudo, aunque sólo una vez se canta un huayno en la cárcel, hacia el comienzo de la novela:

Palomita blanca, palomita blanca
cuculi;
de noche yo vengo a verte
porque de día no puedo,
cuculi madrugadora.

Cámac cantó despacio, con muy débil y delgada voz. -¿y tú?- me dijo.
Torcaza a dónde vas con apresurado vuelo baja y calma mi vida que en triste dolor subsiste.

-¡Eso! Manos a la obra. (El Sexto 50)

Se trata de un momento de alianza entre dos personajes principales, ambos serranos: Cámac y el narrador. Ellos entienden su canto, y sobre todo la guitarra que es el objeto del proyecto que mentan, como una protección ante la maldad de la población limeña carcelaria. Ésta también se expresa a través de la música, pero una que viene del extranjero:

Revista Iberoamericana, Vol. LXXXI, Núm. 253, Octubre-Diciembre 2015, 1051-1064 
Iba a contestarle [a Cámac]. La voz de Rosita interrumpió.

\author{
Partiré canturreando \\ mi poema más triste \\ le diré a todo el mundo \\ lo que tú me quisiste
}

-El marica está con melancolía - dijo Cámac. (El Sexto 51)

La canción de la Rosita, un travesti que habita el primer piso, es un son cubano. La selección de su música, sin embargo, no colma lo que constituye su interrupción. Ella se define por esa música foránea, por cierto, pero también por el hecho de que una figura sexualmente perversa, según la novela, la entone. La novela, además, enfatiza la identificación entre la Rosita y Lima.

Ante la configuración de las relaciones sociales en la cárcel, hay que preguntar ¿a qué responde la creación de la música serrana y por lo tanto, qué significa la música de Rosita? Es la sociabilidad que preocupa a Arguedas aquí, en sus dimensiones éticas, culturales y políticas. Más allá de la degradación moral aguda que existe en El Sexto, podríamos decir que por una parte la sociabilidad se da a través de, o se reemplaza con, las negociaciones económicas. Se trata pues de un sistema económico que encuentra su máxima expresión en la venta de los hombres mismos, en su prostitución. La música que llega a través del mercado de consumo internacional parece grabar no solo su letra en los hombres del Perú; sino también su manera de circular, es decir, la dinámica misma del consumo.

El Sexto se ubica afuera del sistema económico monetario y hasta capitalista, pero en su perfección de la degradación del hombre opera de manera paródica hacia esa economía en el texto. Se venden los cuerpos de los más débiles, como el muchacho Clavel, para abastecer la demanda que produce el deseo sexual y, no está demás decir, la necesidad de dominar al otro. No se pueden separar estos dos deseos en este contexto, pues la jerarquía y la desigualdad de poder que reflejan lo son todo en el Sexto. Además, los cuerpos que se venden tienen más valor que la plata -ínfima- que los compra. Es decir, la cárcel se vuelve ese espacio que permite entender de forma trágica el efecto que la comodificación del hombre tiene sobre la humanidad. El hombre se cosifica, pero no sin resaltar lo perdido en esa transformación.

El resultado de esta cosificación es la degradación extrema. Acaso ningún episodio lo demuestre tan claramente como el de la venta de Clavel a los otros habitantes del primer piso. Los presos realizan el acto sexual a través de las rejas de la celda de Clavel, pues ésta está cerrada con llave. La escena se agudiza en su brutalidad sobre todo a través de su estatus de espectáculo: desde los pisos superiores de la cárcel, se puede fácilmente ver la fila de los que esperan su turno y los actos que los presos cometen ( $E l$

Revista Iberoamericana, Vol. LXXXI, Núm. 253, Octubre-Diciembre 2015, $1051-1064$ ISSN 0034-9631 (Impreso) 
Sexto 136-137). Vale la pena señalar que, ante la atrocidad, el narrador Gabriel intenta protegerse con la lectura de dos textos literarios: El Quijote y "Briznas de hierba". Ninguno lo puede distraer de los actos de violación, pues la lectura de ambos queda interrumpida. La única posible solución, también de filiación poética pero ya de otro modo, es la guitara de Cámac.

Entre los presos políticos, la sociabilidad carente se expresa de otra forma, menos horrorosa pero igual de paralizante: el conflicto ideológico. Los apristas como los comunistas se libran a una lucha ideológica pura, por así decirlo, porque no se contamina con las ganancias, el estatus, el poder, ni sus ventajas, como acaso ocurriría en la sociedad civil. Pero la pureza de esa lucha resulta descubrir la pequeñez inherente en ambas ideologías políticas: se definen una contra la otra, o sea, la esencia de tanto los apristas como los comunistas consiste en la negación del otro grupo y no necesariamente en un acercamiento a la realidad peruana. Curiosamente, y aunque los presos políticos no se degradan físicamente entre sí como los avezados, su violencia epistemológica es absoluta: Gabriel, y cualquier lector, nota que los personajes que encarnan la fe aprista y la fe comunista, también son los que menos oyen. No existe la comunicación entre ellos, pues el odio toma su lugar. Quien conoce la obra de Arguedas conoce la importancia del odio en el diagnóstico que el autor hace de la sociedad andina.

Entonces, ¿qué tipo de sociabilidad permite pensar la forma privilegiada del huayno? Creo que la novela plantea dos opciones, ligadas pero de índole distinta. La primera acaso se exprese más nítidamente a través de los huaynos en quechua o español que Gabriel y Cámac suelen rememorar. Tres ejemplos: Cuculí madrugadora (50), Yana k'enti (60), o Mayra rinki yurak paloma (118). Ellos manifiestan la cultura andina y quechua como una defensa, pues suelen movilizar la historia personal y compartida como componente vital de una sociedad, también andina, que se valoriza por su persistencia y solidez, y por lo tanto por su capacidad de darle forma al mundo. Se trata de una cosmovisión. En cierta medida, estos huaynos transforman la nostalgia en respuesta ante los desafíos que representa la sociedad nacional y criolla. La sociabilidad que se figura en esta primera instancia sigue siendo fuertemente marcada por la sierra como lugar de origen y sentido.

Pero la sociabilidad se figura de otras maneras también. El papel clave de la música se manifiesta de forma tajante en la conclusión enigmática de la novela. Pero allí se resalta a través de su ausencia. Luego de una afirmación que la degradación que sufren los presos constituye una meta de la institución y no simplemente ocurre como casualidad en la vida del Sexto, el piurano, aliado del narrador Gabriel, mata al soplón conocido como el Pato, uno de los jefes del Sexto. La noticia la difunde Gabriel mismo, en una representación admirable respecto de las posibilidades de la oralidad, en castellano:

Revista Iberoamericana, Vol. LXXXI, Núm. 253, Octubre-Diciembre 2015, 1051-1064 
Entonces grité yo, corriendo al primer puente.

-¡Señores, compañeros! El piurano acaba de degollar aquí al Pato. ¡Viva el piurano! Esperé la respuesta, largo rato, en el puente, contemplando las puertas de las celdas. Nadie, ni Mok'ontullo ni Torralba contestaron. El guardia se arrepintió de abrir la gran reja. Empecé a distinguir, puerta a puerta todas las celdas, hasta el fondo. ¡Era otra vez un cementerio! ¡Más que un cementerio! Los vivos estaban muertos. Los entonadores de los himnos a cuyo fuego don Policarpio [el piurano] extrajo como un rayo su cuchillo y le rompió el cuello a uno de los soplones más temibles de Lima, estaban muertos. Escuché un murmullo sordo en el piso de los vagos. Recordé toda la melodía y la letra del canto fúnebre con que en mi pueblo enterraron a ese desconocido, que llegó con un lorito en el hombro y cubierto con un poncho negro, de rayas amarillas que parecían hechas de luz. ¡Cómo cantaron las mujeres bajo la inmensa sombra de las montañas, en el andén del cementerio! Iba a empezar ya el canto:

"Oye, negra flor de pensamiento..." (El Sexto 150)

Luego de una pausa, y antes que Gabriel pueda cantar el huayno en quechua cuya traducción cierra la cita previa, se escucha el canto de los prisioneros políticos. Éste ocurre en dos episodios, que se turnan:

Pero Luis gritó, con voz enérgica y delgada:

-¡Compañeros: nos dicen que el piurano ha degollado al más feroz chacal del Gobierno!

¡Viva el piurano!

-¡Vivaááá! -le contestaron centenares de hombres.

-iViva el apra!

-¡Vivaááá!

Y luego la voz de Pedro:

-¡Camaradas!: el campesino piurano Policarpo Herrera ha liquidado al feroz verdugo el Pato. ¡Viva el piurano!

-¡Vivaááá!

-¡Viva el Perú!

-¡Vivaááá! (El Sexto 150)

Es la reacción de Gabriel a esta escena, en voz alta, que me devuelve a mi pregunta inicial: "Me oyeron, solamente. Yo seguiré haciendo la guitarra, hermano Cámac" (El Sexto 151).

La sentencia de Gabriel, que además comunica la meta que definirá su vida, nos revela las potencias divergentes de la oralidad verbal y la sonoridad musical. La comunicación en castellano fracasa al no poder difundir el conocimiento deseado, y resulta ser la música, que todavía no se puede tocar, la que podría hacer efectiva la comunicación incompleta. La música como máquina para interpretar se encarna aquí pues, literalmente, en la guitarra inconclusa de Cámac; pero habría que hacer la pregunta: ¿Máquina para interpretar qué exactamente?

Revista Iberoamericana, Vol. LXXXI, Núm. 253, Octubre-Diciembre 2015, 1051-1064 
Por otra parte, de forma abstracta, la música viene a sugerir otra posibilidad, ya no en el pasado sino en el futuro. Justamente, en el pasaje que he citado es la audición sin entendimiento de las palabras de Gabriel que comunica la necesidad para una nueva musicalidad, que exceda la nostalgia pero también los límites de la cultura rural in situ. Por ello, esta música nunca se escucha en El Sexto. Se trata pues de la guitarra inconclusa de Cámac, que además de materializar la cultura serrana también resulta ser el símbolo de otra sociabilidad que no termina de definirse.

A pesar de su ausencia en la novela, quiero sugerir una pista para conceptualizar lo que podría ser esta música no realizada. Siempre me ha parecido más que curioso que el momento en que Arguedas escribe la gran parte de sus novelas coincida con el auge del huayno en el mercado del consumo musical nacional. La industria disquera de música andina está en pleno desarrollo a mediados de siglo XX, y Arguedas no solamente conoce ese fenómeno, sino que también dedica parte de su obra ensayística a dicha música, y además promueve activamente algunos de las estrellas del huayno, como las hermanas Zevallos y el Jilguero del Huascarán. Está pues en contacto íntimo con la transformación del huayno, que suele representar en su manifestación más tradicional en sus novelas. En sus escritos periodísticos, Arguedas atestigua asiduamente el surgimiento del huayno masivo, costeño, y migrante, que se escucha a fuerza y luego por preferencia en canciones cernidas a los límites de tiempo y forma que la tecnología y la coyuntura urbana nueva dictan, en long play y en estadio, en quechua y en español, y claro está en la ciudad de Lima, que comienza a volverse otra.

Sospecho que la resistencia a representar el presente y el futuro de la música andina que se hace palpable en la guitarra inconclusa de Cámac tiene que ver con esta realidad. Hay un peligro en este contexto de urbanización y tecnologización de la cultura andina, que pienso aparece representada en El Sexto también. Por ejemplo, la degradación extrema del Clavel se expresa así: "Afuera pudimos percibir la letra de los cantos [del Clavel]. Eran huaynos que [Clavel] mezclaba con letras de tangos y rumbas [...]" (93). Gabriel y Cámac reaccionan con pena ante la canción del violado, pero también con la certeza de que esa mezcla significa la locura, y por lo tanto la degradación absoluta. Es el conocido temor a la contaminación que aparece a menudo en la obra de Arguedas, y que suele expresarse a través de la agresión sexual. Propongo que por eso queda inconclusa la guitarra de Cámac: su estatus incompleto constituye el reconocimiento de la posibilidad que la cultura andina se mezclará, se transformará, acaso hasta el punto de no ser reconocible, y que esa transformación resulte necesaria ante lo que significa el contexto de la ciudad. Arguedas resiste representar la música en su transformación consumista, pero sí importa a su novela la funcionalidad del nuevo huayno, no oído: volverse música de la modernidad, del acá y del aquí, acaso para nosotros y para ellos. Se trata pues de otra instancia arguediana de cavilar la cultura andina en la modernidad, vale decir, en la coyuntura urbana, consumidora, y heterogénea.

Revista Iberoamericana, Vol. LXXXI, Núm. 253, Octubre-Diciembre 2015, 1051-1064 
Si bien la guitarra de Cámac no logra asumir una forma definitiva en El Sexto, ese esfuerzo continúa en la obra ensayística de Arguedas que trata la música en el ambiente urbano. ¿Cuál habría sido la banda sonora de la ciudad de Lima hacia los años 1930s? Sin duda, en ella habría sido notable la preponderancia del vals limeño, pero también del tango argentino, el fox-trot, y otros ritmos recientemente allegados al territorio nacional. Es difícil imaginar en ese momento oír en los barrios de Lima música serrana, el huayno, la muliza, o el yaraví por ejemplo. Sin embargo, la población andina urbana ya crecía por esos años, y entonces la compenetración de la ciudad con el huayno no se haría esperar, al menos no mucho. A partir de la década del 50, ya se podía contar con una banda sonora variada y ubicua. Según un equipo de investigación de la Universidad San Martín de Porres, en 1954 Lima contaba con diecisiete radioemisoras y doce años más tarde, en 1966, ya habían 89 en la costa. (Universidad de San Martín de Porres 88)

No sería inverosímil, desde ya, demostrar en el momento histórico que retrata $E l$ Sexto una música reproducida mecánicamente, pero la presente no es una preocupación con lo que se representa en cuanto a la verosimilitud del ambiente musical en esa novela. Más allá de la representación directa, me preocupan los modelos plásticos culturales que la música puede asumir, es decir, cómo se puede absorber, o no, la idea o el uso cultural de la música en un momento histórico dado, a través de la novela. Si bien la representación de la música funciona para interpretar los Andes dentro del momento moderno, también sirve para entender el impacto del mercado de consumo de bienes culturales en ese mismo momento. Por esto, precisamente, se resalta la producción artesanal -es decir, no industrial- de la guitarra de Cámac.

Aunque hay muchísimos ejemplos en la obra ensayística, bastarán dos instancias para iluminar la función doble de la música en cuanto aparato cultural interpretativo. Curiosamente, y aunque los ejemplos disten en años, ellos se repiten a lo largo de la vida productiva del autor, ya sea antes o después de El Sexto. Propongo que constituyen un eje central en la teorización de la cultura en la obra de Arguedas. Como dijimos al comienzo del presente ensayo, se trata de cómo la música se usa para interpretar. En ambos casos, como en la novela, se trata de la sociabilidad que esta música genera o facilita, pues más allá de su uso relativamente simple en cuanto a la preservación de las formas culturas andinas, también funciona para generar nuevas prácticas, tan potentes como las antiguas.

Interesantemente, ambas instancias tienen su escena en Lima. Ya en 1944, Arguedas escribía con agudeza respecto a los peligros del mercado y sus efectos deformadores sobre la cultura andina. "En defensa del folklore musical andino", apareció en el periódico limeño La Prensa en noviembre de 1944. La fecha temprana indica la reflexión detenida del autor respecto a la suerte de la música andina en relación a la nacionalidad. Claro está que esto lo constata también, y acaso con mayor complejidad, su obra narrativa posterior. El artículo se enfoca en la cantante Ima Sumac, que por

Revista Iberoamericana, Vol. LXXXI, Núm. 253, Octubre-Diciembre 2015, 1051-1064 
esos años comenzaba ya a tener un renombre nacional e incluso internacional. Nacida en El Callao y bautizada como Zoila Augusta Emperatriz Chávarri del Castillo, Sumac encarna para Arguedas la falsificación de la música andina a favor del espectáculo rentable. Luego de asistir a un concierto suyo en Lima, Arguedas juzga:

¿Cómo podía pretenderse que cantara las canciones andinas quien no sabía una sola palabra de Quechua? Una joven que había crecido en Lima cuya psicología había sido muy modelada bajo la influencia humana total de los barrios de Lima, sin ninguna cultura musical, no podía estar en condiciones más negativas para pretender convertirse en intérprete de la música india. ("En defensa” 234)

Arguedas señala la incoherencia entre el origen citadino de la cantante y el acervo cultural serrano al que se acerca. Sin embargo, éste no parece ser el problema principal. Ése está, más bien, en lo que motivó el fenómeno que encarna Ima Sumac: "[deformar] la canción andina quechua hasta hacerla accesible al sentido superficial, frívolo y cotidiano del público de la ciudad" ("En defensa" 234). Lo intolerable es pues la separación de la forma andina de su intimidad tradicional o esencia cultural para circular como otro bien, vale decir, de consumo. Por lo tanto, los efectos deformadores no tienen simplemente el efecto de malograr la tradición andina, es decir, de traicionar su forma y contenido histórico. Más bien, el mayor peligro yace en perder su capacidad formativa y progresista. Sin ella, lo andino no tiene validez para el futuro pues es incapaz de moldearlo.

El segundo ejemplo toma particular resonancia a la luz de El Sexto. En el artículo "De lo mágico a lo popular, del vínculo local al nacional," publicado en el suplemento dominical del periódico limeño El Comercio en junio de 1968, Arguedas de alguna forma trata de entender las posibilidades redentoras del mercado de bienes culturales. Como lo haría en otros artículos en los cincuenta y sesenta, Arguedas nutre su pensamiento de la actividad de los coliseos de Lima y la cultura popular pululante que allí se encontraba. Arguedas los describe así:

En el Perú la palabra "coliseo" nombra ahora a los locales abiertos o techados de carpas de circo, en los que se ofrecen programas de danzas y músicas folklóricas andinas, cada vez más frecuentemente matizadas de música criolla y latinoamericana. Los "coliseos" son locales rústicos, "humildes", acogedores, decorados en sus fachadas y escenarios con figuras "incaicas" espectaculares o ingenuas. El "coliseo" atrae al campesino temeroso aún de los secretos de la ciudad, al criollo de barrio o barriada, al hombre ilustrado y sensible. ("De lo mágico" 244)

Arguedas se preocupa por subrayar la mezcla o mistura, como él decía, que fomenta el fenómeno del coliseo. Queda claro aquí que lo que se aprecia del coliseo no es simplemente su capacidad de integrar clases y culturas peruanas que divergen

Revista Iberoamericana, Vol. LXXXI, Núm. 253, Octubre-Diciembre 2015, 1051-1064 
y hasta se contradicen en otros espacios. La ubicación periférica de los eventos en la ciudad de Lima, y además también en la provincia, es una parte crucial de la plasticidad potente que las define. Es más, Arguedas se enfoca en dos aspectos que resuenan en $E l$ Sexto: la suciedad y el gentío, que se describe como "denso y agitado" ("De lo mágico" 244). La cárcel también, aunque ya en mayor grado y con efectos más degenerativos, contiene estas características. Es decir, y aunque ella esté en medio de la ciudad, es un espacio de límite o de periferia y también resulta ser un espacio que acumula hombres distintos que posibilitan una mistura extrema. El fenómeno del coliseo ha sido estudiado con detenimiento por el crítico Javier García Liendo.

El personaje central del artículo sobre los coliseos es un afroperuano llamado Juan Aguilar, conocido como el Gavilán Negro. Se lo describe de la siguiente manera:

El joven negro fue cautivado por las orquestas huancas y la música del Mantaro... El mozo negro... memorizaba, domingo a domingo, directamente de las orquestas y cantantes, las melodías huancas; ensayaba, primero a escondidas, en el propio escenario del coliseo vacío, en los cuartos y pasadizos del local, los pasos de los bailes, el huaylas, la muliza, el huayno, hasta la elegante "Chongueada." Escuchaba los programas de música serrana que ofrecen las radiodifusoras, al amanecer, y a Radio Agricultura que dedica nueve horas diarias al folklore andino. Cuando se sintió preparado, de ánimo, voz y danza, para actuar, pidió a uno de los directores de la orquesta huanca que lo probara. Los artistas estimaban al siempre bienhumorado "moreno" [...] Eligió "Vil cocodrilo" para cantar, y unos minutos después, se agolpaban en la puerta del camerino decenas de artistas sorprendidos y curiosos. (244-47)

La cita y el texto del que proviene ofrecen una riqueza extraordinaria respecto a cómo entendía Arguedas la inserción de la música andina en la ciudad. Aquí vale la pena señalar una diferencia esencial entre el acercamiento a Ima Sumac y a los coliseos, y sobre todo lo que esa diferencia señala en cuanto al entendimiento del fenómeno cultural. Hemos visto que en el primer caso hay una distancia decisiva entre la practicante y la tradición cultural a la que (malamente) se refiere; en el segundo caso, aunque existe la misma distancia entre Juan Aguilar y la música serrana, existen condiciones para que esa brecha se pueda cerrar. A diferencia de la polarización que ocurre en El Sexto, se percibe claramente que la mistura del afroperuano con las costumbres andinas no solamente es posible, sino también resulta culturalmente fértil. De alguna forma, el coliseo resulta ser la continuación del mismo proyecto que se cifra en la guitarra de Cámac. Ya para 1968, la máquina sí permite interpretar, de manera rotundamente exitosa, lo andino dentro de la ciudad moderna. Arguedas claramente entiende así el coliseo, pues allí: "Costa y sierra se funden a fuego, se integran, se fortalecen" ("De lo mágico" 247).

Revista Iberoamericana, Vol. LXXXI, Núm. 253, Octubre-Diciembre 2015, 1051-1064 


\section{BIBLIOGRAFÍA}

Arguedas, José María. "De lo mágico a lo popular, del vínculo local al nacional". Señores e indios: acerca de la cultura quechua. Ángel Rama, ed. Buenos Aires: Arca, 1976.

"En defensa del folklore musical andino". Señores e indios: acerca de la cultura quechua. Ángel Rama, ed. Buenos Aires: Arca, 1976.

Katatay. Lima: Horizonte, 1984.

Los ríos profundos. Madrid: Cátedra, 1958.

Señores e indios: acerca de la cultura quechua. Ángel Rama, ed. Buenos Aires: Arca, 1976.

El Sexto. Lima: Horizonte, 2011.

Beasley Murray, Jon. "Arguedasmachine: Modernity and Affect in the Andes." Iberoamericana 8/30 (2008): 113-128.

Cornejo Polar, Antonio. Los universos narrativos de José María Arguedas. Lima: Horizonte, 1997.

García Liendo, Javier. "Las chicherías conducen al coliseo: José María Arguedas, tecnología y música popular". Revista de crítica literaria latinoamericana 38/75 (2012): 149-70.

Rowe, William. Ensayos arguedianos. Lima: Sur, 1996.

Salazar Bondy, Sebastián. Lima la horrible. Lima: PEISA, 1974.

Universidad de San Martín de Porres. Escuela Profesional de Turismo y Hotelería. Instituto de Investigacíon. El Impacto Económico de La Cultura En Perú. Lima: Convenio Andrés Bello, 2005.

Revista Iberoamericana, Vol. LXXXI, Núm. 253, Octubre-Diciembre 2015, 1051-1064 